Sharif University of Technology
Scientia Iranica
Transactions E: Industrial Engineering
http://scientiairanica.sharif.edu
IRA I ENTIA

\title{
An accurate analysis of the parameters affecting consumption and price fluctuations of electricity in the Iranian market in summer
}

\author{
S.M. Kavoosi Davoodi ${ }^{\mathrm{a}}$, S.E. Najafi ${ }^{\mathrm{a}, *}$, F. Hosseinzadeh Lotfi $^{\mathrm{b}}$, and \\ H. Mohammadiyan Bisheh ${ }^{\mathrm{c}}$ \\ a. Department of Industrial Engineering, Science and Research Branch, Islamic Azad university, Tehran, Iran. \\ b. Department of Mathematics, Science and Research Branch, Islamic Azad university, Tehran, Iran. \\ c. Department of Industrial Engineering, Mazandaran University of Science and Technology Branch, Babol, Iran.
}

Received 19 January 2019; received in revised form 22 January 2020; accepted 13 July 2020

\section{KEYWORDS \\ Energy prediction; Hybrid network; Evolutionary search; Data analysis; \\ Deep neural network.}

\begin{abstract}
In this paper, a novel method is proposed to predict the cost of short-term hourly electrical energy based on combined neural networks. In this method, the influential parameters that play a key role in the accuracy of these systems are identified and the most prominent ones are selected. In the proposed method, initially, using the Self-Organizing Map (SOM) network, similar days are placed in close clusters. In the next stage, due to their differences in the scope and nature of their changes, the temperature parameters and prices related to similar days are trained separately in the two Multilayer Perceptron (MLP) neural networks. Finally, the two networks are merged with another MLP network. In the proposed hybrid method, an evolutionary search method is used to provide an appropriate initial weight for neural network training. Given the price data changes, the price amidst the previous hour has a significant effect on the prediction of the current state. In this vein, in the proposed method, the predicted data in the previous hour is considered as one of the inputs of the next stage. The proposed method was assessed on the datasets of Iran in the summer. This information pertains to the 2011-2016 period.
\end{abstract}

(C) 2021 Sharif University of Technology. All rights reserved.

\section{Introduction}

Since the industrial revolution, energy has become a key factor in everyday life [1]. Fossil fuels have gradually become the most important energy source. [1]. However, with population growth and technological development, the contemporary world faces two major problems: environmental pollution and energy short-

*. Corresponding author.

E-mail address: Najafi1515@yahoo.com (S.E. Najafi) age [2]. One way to overcome problems is to improve efficiency and reduce emissions [3]. The other way is to develop alternative energy resources [2]. People are looking at renewable resources because they are environmentally friendly and sustainable. The most competitive renewable energies include water, wind, photovoltaic energy, and biofuel. Many of them have been proven to be advanced in solving environmental and energy problems [4,5]. Today, electricity, as one of the most popular types of energy, has become a basic element of human life. In order to supply this energy, vast and extensive power systems have emerged in various countries. If they were assigned to the 
private sector, they would have a vertically integrated management structure. The management and control of such systems was initially the responsibility of governments or quasi-public institutions, and if assigned to the private sector, they would have a vertically integrated management structure. Most of the previous theories presented about power systems were based on the idea that electricity is a public service sector with intrinsic monopolistic properties. Many renewable energy sources have been used in the electricity market. In the past few years, as the marginal cost of renewable energy was close to zero, the electricity market price has fallen sharply [6]. Therefore, the electricity market participants are seeking ways to be more competitive in the market. Regarding electricity price, many companies have adopted new tariff plans [7], such as time-of-use tariff plans. These plans charge higher rates when demand is high, and lower rates when demand is low. This encourages customers to make wise decisions about their electricity consumption and reduce peak energy usage [8]. This situation makes not only the producers but also the customers pursue more precise forecasts of the electricity market prices than ever. However, electricity price usually has complex features, such as highly volatile behavior and nonlinearity, which makes it rather difficult to build a precise forecasting model [9-10].

Accurate electricity price forecasting may help electricity market participants to formulate reasonable competition strategies. Specifically, power producer scan use the forecasting results to optimize unit output, while power consumers can use the results to optimize purchase portfolio [11].

However, the complex features of electricity prices such as periodicity and high volatility make the forecasting pretty difficult [12].

In recent years, a lot of models have been proposed for electricity price forecasting $[13,14]$. In general, the commonly used models can be classified into two primary categories: soft computing models $[15,16]$ and time-series models $[17,18]$

The amount of information available to participants in the market is a fundamental issue in selecting the type of method used to resolve the pricing issue. Since the competitors in the electricity market do not adopt a specific predictable procedure because of the market competitive nature, producers' behavior in pricing strategy regulation is not logical and the assumption of profit maximization is not rational to predict competitors' behavior. Thus, the application of the game theory and smart algorithms is not appropriate due to the restrictive hypothesis in problem modelling, such as predicting competitor power plant costs and the rationality of their behavior in setting pricing strategies.

The factors that influence the prediction of elec- tricity prices are: 1) Weather conditions, seasons, daytime and nighttime, 2) History of electricity price data, 3) Demand and production balance, 4) Market participants' strategies, 5) Fuel prices, 6) Transmission lines congestion, 7) Market design, and 8) Time of power cuts from main power plants. The presented solutions can be grouped based on the applied methods or schemes. It is common practice to implement Short-Term Price Forecast (STPF), Medium-Term Price Forecast (MTPF), and Long-Term Price Forecast (LTPF). Depending on the selected prediction method, the input and output of the model can vary. According to the literature, the input of models may be as follows: load history, storage history, heat, production capacity, line constraints, predicted load, predicted storage, weather, oil prices, gas prices, input fuel, price history, bidding strategy, winter index, summer index, possible transmission line information, density index, holidays code and type of day (holiday or nonholiday) [19]. The output of various models can also be of the following type: price profile, quantity profile, load profile, maximum price, the average price at peak load time, average error, and average error percentage. The pricing issue is an optimization issue. When the objective is to solve an issue in the real environment, the precise conditions of the issue must be considered.

In the field of market restructuring and privatization, the price factor and its accurate prediction are of increasing significance. Thus, according to the aforementioned issues, the purpose of this research is to answer the following question: What is the optimal strategy for predicting the price of electricity in a competitive electrical energy market within the framework of the laws and regulations of the Iranian electricity market?

In this regard, in the present research, the following hypotheses will be used to explain the utilized approaches:

Among the types of power plants, only the steam power plant is examined. In determining the optimal pricing strategy, the prediction of the next day load is considered definitive.

However, to demonstrate load variances in the results, sensitivity analysis is conducted for load variations.

The electricity market, as with all other markets, will be a demand-driven market. As with the real electricity market, the supply suggestion function in this market will be linear and ascending relative to the production level, and the demand function will also be linear but descending relative to the demand level.

To determine the optimal price strategy, the DC load distribution is used in the market pricing model.

For instance, Panapakidis and Dagoumas [20] used the Artificial Neural Networks (ANNs) model for electricity price forecasting in Southern Italy. Sandhu 
et al. [21] employed neural networks to forecast Ontario electricity prices. To better capture the characteristics of electricity prices, a combination of ANN models and other models is often presented. For instance, Ortiz et al. [22] proposed a combined model based on ANNs. Keles et al. [23] developed a model based on ANNs and an optimal parameter model. Singh et al. [24] presented a model that combines a generalized neuron model with the wavelet transform. Itaba and Mori [25] utilized the general radial basis function network and fuzzy clustering. Wang et al. [26] developed a hybrid model combined with ANNs and decomposition technique. It should be noted that although the ANNs model can describe the nonlinear characteristics of electricity price series, it cannot well deal with the linear fitting problem [27]. To describe the linear features of electricity prices, the time series model is often applied, which is considered one of the most effective techniques [28]. Traditional time series models, such as Autoregressive Integrated Moving Average (ARIMA), Autoregressive and Moving Average (ARMA), and Generalized Autoregressive Conditional Heteroscedasticity (GARCH) have been frequently applied to forecast electricity prices. Besides, Diongue et al. [29] and Girish [30] proposed some new time series models such as GIGARCH and autoregressiveGARCH. To better capture the features of electricity prices, some other models have been combined with time series models $[31,32]$. Since the electricity price series is composed of linear and nonlinear components, the integrated models with linear and nonlinear fitting capabilities can improve the forecast accuracy $[33,34]$. For this reason, the Empirical Mode Decomposition (EMD) approach has been used for electricity price decomposition by some researchers $[35,36]$.

\section{Research methodology}

Since the predictions in this study occurred in Iran with specific climatic conditions, the aim was to use new parameter combinations and more accurately classify the data into various categories. Parameters such as past load prices, temperature, and humidity in each category are considered through the new combination of these features. Data categorization methods and the precise selection of parameters are discussed further.

In this project, information on electricity load prices in Mazandaran Province from 2014 to 2017 was used, and the temperature during this period was taken into account. In the data collection stage, the data is analyzed and dynamically identified, so that the data is divided into smaller groups based on their common characteristics, and a separate model is created for each group. In numerous sources, it is emphasized that various pricing activities cannot be presented by one model.
In this case, initially, without considering the specific data classification, the consumed loads during the defined period were predicted, but there was no favorable result. Given the substantial changes in electricity consumption amidst season changes, categorization should first be conducted based on seasons. Hence, data are categorized into four groups, i.e., spring, summer, fall, and winter. Nevertheless, it should be pointed out that, except for summer, forecasts for all seasons are conducted accurately by current systems and human expertise.

Different days of the week have their own curves, although in previous studies it was claimed that the curves for mid-week consumed loads (for Iran, from Sunday to Wednesday) are similar to but different from the load curves for holidays. In addition, the consumed load curve for the days before and after the holiday is different from the normal number of days in the week. However, in the real world, it is not possible to manually conduct such categorization. Therefore, this paper presents a categorization method to schedule these cases.

One of the other factors impacting the consumption price curve is the holidays. Since there are two types of holidays in Iran, i.e., religious and national holidays, both should be taken into consideration. In this project, forecasts of religious and national holidays are conducted separately. However, it should be noted that, according to the comparison of consumed load prices from 2009 to 2012 , the consumed load in Iran and expert opinions indicate that some holidays do not need to be forecasted in terms of consumed loads because the loads consumed these days are similar to previous years. For example, the consumed load on 2nd April and Ashura Day are the same as the previous year.

\section{Hybrid neural network}

ANNs are suitable tools for modeling and forecasting data. Various types of neural networks have been introduced, each with a specific application. One of the main and beneficial functions of neural networks is their effect on a large number of variables and complex systems. Despite the simplicity of utilizing neural networks, there are also drawbacks such as setting network architecture parameters, placing the network in local optimizations, and extension of the learning process time period. In this regard, various solutions have been proposed to solve each problem, and one of the most advantageous is to combine these networks. The combination of neural networks varies in different applications. One of the most valuable functions of this type of network is the use of a non-monitored neural network to cluster similar data, and in the next stage, to train the supervised networks using similar samples in one cluster. Another use of the hybrid network is 
that the input range is different, which leads to the lack of proper network training and the negligence of some traits. In this regard, different types of traits are trained with different networks, and these types of networks are finally combined.

The reason for utilizing hybrid networks to forecast the price of the consumed load is the existence of several effective factors. Due to the lack of inaccessibility to all of these factors, two of the most valuable features, i.e., temperature and cost of previously consumed loads are used. These two parameters are highly influential on one another but, at the same time, are extremely different in nature. The most important reasons for this include:

1. Temperature difference of a few degrees may multiply the consumed load price by a few hundred. Therefore, the slightest temperature changes in the network should be accurately modelled;

2. If the consumption load of the assessed province is between 200 and $1500 \mathrm{MW}$, the range of temperature changes is within 5-30 degrees Celsius;

3. The effect of temperature variation in different hours of the day exhibits a different trend. As an example, a temperature change of two degrees between the hours of 13 to 15 shows a significantly greater impact on consumption compared to a twodegree change in the early hours of the day;

4. Temperature changes in various seasons do not exhibit the same effect. For example, a one-degree temperature change in summer exhibits a different change compared to the same change in winter;

5. Temperature changes within temperature ranges are also significant. For example, a temperature variation of two degrees within a temperature range of fewer than 20 degrees may not exhibit much effect on load consumption but a temperature variation of one degree at temperatures over 24 degrees will exhibit a significant effect on load consumption.

As previously mentioned, these parameters are of a completely different nature in terms of size but are highly influential on one another. For this reason, two different networks have been used in this project for these two parameters.

\section{Self-organizing map neural network}

The Self-Organizing Map (SOM) neural network (SOM) is an unsupervised network for clustering data. In each application to the input, the algorithm maps its self-organizing Kohonen features to neurons from onedimensional or two-dimensional network type neurons. This type of neural network is organized by input samples, and finally approximates the distribution of network inputs in a discrete environment. This network consists of two layers: The first layer is the input layer, in which input samples are inserted and applied to the network neurons through it. The second layer consists of the output neurons. In a normal state, each neuron has only one binary output possessing a value of one or zero. If the neuron in question wins the resource competition, its output will get a value of 1 , and the output of the remaining neurons will be zero. The neuron that weight has the most resemblance to the input sample is considered the winning neuron for specific input. In this case, its output gets a value of 1 , and the output of the remaining neurons will be zero. The weight vector of the winning neuron is corrected along with its neighboring neurons. This correction causes the progression of the neurons' weights towards the recent input, whilst the weights of the other neurons will remain unchanged.

This is one of the most important parameters for detecting the number of clusters (the number of similar days for this project). For this purpose, the k-mean and Fisher hybrid algorithm is used for this project.

\section{Genetic algorithm}

A genetic algorithm is a programming technique that uses genetic evolution as a problem-solving model. The input is the problem to be solved and solutions are coded according to a pattern called the fitness function that evaluates each possible solution most of which are randomly selected.

Genetic algorithm is a search technique in computer science used to determine the optimal solution and solve the search problem. These algorithms are a type of evolutionary algorithms inspired by biological science branches such as heredity, mutation, saltation (biology), natural selection, and composition.

Evolution starts from a completely random set of entities and is repeated in subsequent generations. In each generation, the most suitable ones are selected instead of the best. Three criteria are typically used as stopping criteria: 1) runtime of algorithm 2) number of generations, 3) convergence error criteria.

The most prominent applications of the genetic algorithm include hydrological routing of runoff in a dry river network, assistance in resolving multi-criteria decision issues, multi-objective optimization in water resources management, optimization and loading of electricity distribution networks, etc.

\section{Selection of input parameters and variables}

Based on previous evaluations, time and temperature variables were selected as the two influencing factors that affect the price and previously consumed loads. Among the climate variables, only the temperature 
variable is used since most other climate factors are included in this variable. Given the fact that temperature has a significant influence on the consumption trend, namely in the northern region of the country, and since the forecast is calculated on an hourly basis, it is obvious that the use of temperature parameters based on an hourly or closer time interval will require improved operations. It is important to consider the previous number of days and weeks in terms of the consumed load price and temperature. According to studies and the opinions of electricity distribution experts, the use of load and temperature at various hours of the previous days and weeks is highly effective (although it can be equivalent to the hours of the forecasted day). In this project, data of the two preceding days and two equivalent days of previous weeks were used. In addition, information on the hourly temperature of the forecasted day is also used. Other utilized useful information included the load price and temperature of the preceding hour. Since information on the preceding 24 hours of the forecasted day is available, to forecast the next hours, the data pertaining to the previous hours is used as input.

\section{Proposed method}

A vital factor in the future planning of power systems and electricity market management is the forecast of short-term load price. In recent years, due to the significance of this issue, various methods have been presented to improve the performance of such systems. This paper presents a modern method to forecast shortterm hourly electrical energy costs based on hybrid neural networks. In this method, influential parameters that play a key role in the accuracy of these types of systems are identified and the most prominent ones are selected. Due to the different fluctuations in electricity prices on different days and seasons, these parameters do not follow a common pattern. Therefore, in order to improve forecasts, data is divided into classes that are close in nature. Since one of the effective forecast parameters is the detection of similar successive days in a week, data pertaining to various seasons are analyzed separately. In the proposed method, initially, by using the SOM network, similar days are placed in close clusters. In the next stage, the price and temperature parameters of similar days are trained separately in two Multilayer Perceptron (MLP) neural networks due to their difference in nature and range of changes. Finally, the two networks are merged with another MLP network. In the proposed hybrid network, the evolutionary search method was used to assign a suitable initial weight to train the neural network. Due to the changes in price data, the price of the previous hour has a significant effect on forecasting the current state. Therefore, in the proposed method, the forecasted data of the preceding hour is used as one of the inputs for the next stage. Figure 1 shows an overview of the proposed method.

In the proposed method, the trained dataset is initially allocated to the SOM neural network. In this stage, the number of clusters is determined using the k-means and Fisher criterion hybrid method. This number varies for each hour of the day. Thus, network training for 24 hours is conducted separately and in succession. In the proposed structure, after specifying the number of clusters, it should be determined which cluster each sample entering the network from the training data set belongs to. In the next stage, based on the selected cluster, the associated MLP network is set up. Essentially, each MLP network is trained with the dataset pertaining to its relevant cluster. Finally, for the test dataset, the sample distance from the clusters is first determined along with its relevant cluster. Then, the test sample is evaluated using the associated MLP network to the cluster.

In the proposed method, each cluster not only uses one MLP network but also uses a combination of MLPs. The input data set is divided into two categories: load price and temperature, which are extremely different in nature, and their effects are significantly different due to the range of change. In the proposed method, two separate networks are used for temperature and load. Finally, the outputs of these networks are combined with other MLPs to achieve predictions. In the proposed method, the output for each hour is used as the input for the next hour. The data pertains to the years 2010-2016. In addition to extracting price information, the consumed load and temperature are also extracted in units of hours. In addition to extracting price information, the consumed load and temperature are also extracted per unit of hour. Data analysis is done using MATLAB. In this paper, evaluation criteria such as the Mean Absolute Error (MAE), Mean Absolute Percentage Error (MAPE), and $R^{2}$ are used.

The mean absolute error refers to the difference between the predicted value and the real value which is shown by Eq. (1):

$$
\mathrm{MAE}=\frac{1}{n} \sum_{i=1}^{n}\left|y_{i}-\hat{y}_{i}\right| .
$$

The mean absolute percentage error or MAPE is calculated using Eq. (2):

$$
\mathrm{MAPE}=\frac{1}{n} \sum_{i=1}^{n}\left|\frac{\hat{y}_{i}-y_{i}}{\hat{y}_{i}}\right| \times 100 .
$$

The $R^{2}$ criterion is the statistical measurement of how close the data are to the fitted regression line. $R^{2}$ is also called the determination coefficient of the 


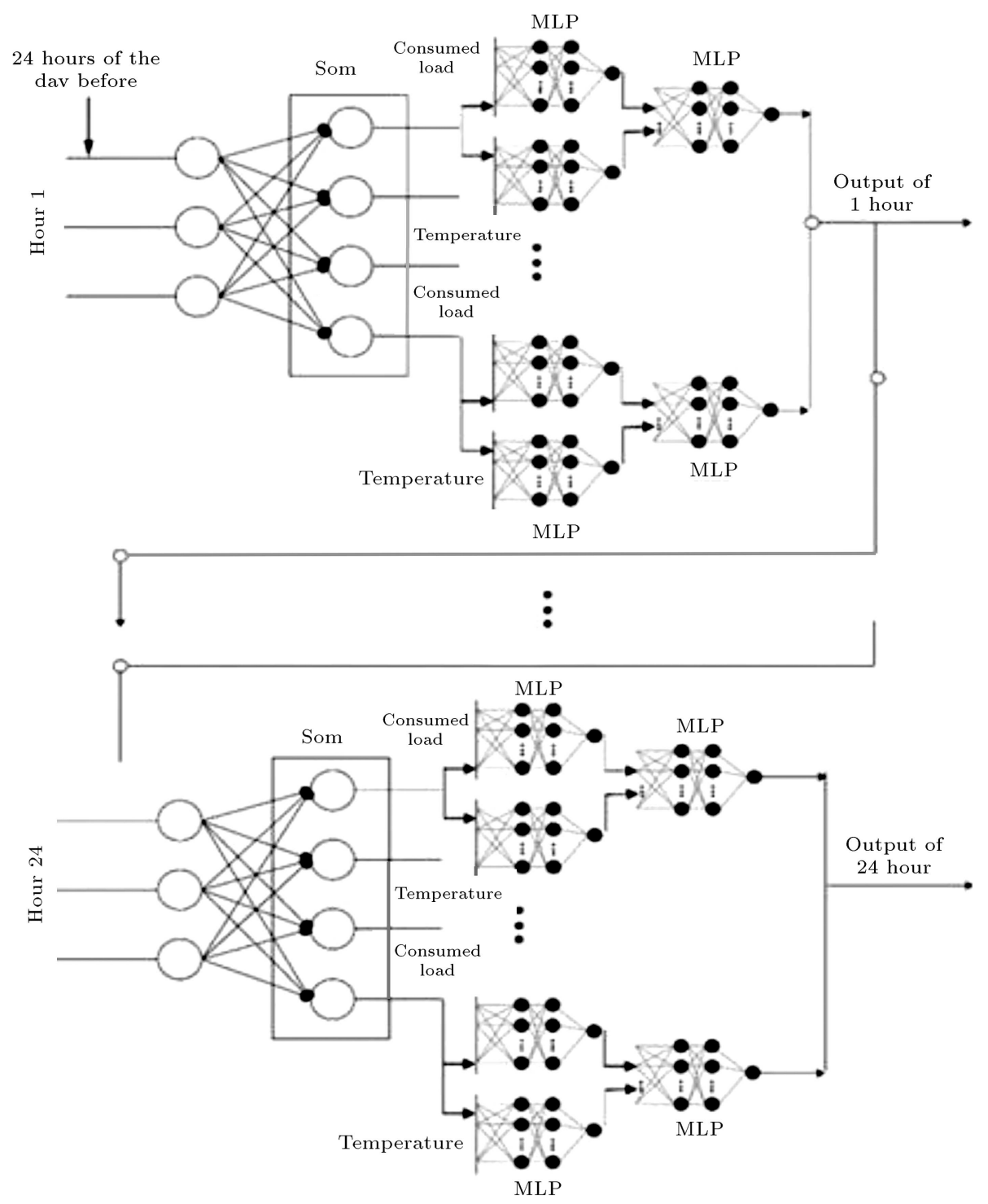

Figure 1. An overview of the hybrid network to forecast the load price of the next 24 hours.

detection coefficient. The definition of the determination coefficient $\left(R^{2}\right)$ is relatively simple: "the determination coefficient $\left(R^{2}\right)$ indicates the percentage of variations of a dependent variable determined by the independent variable" or in other words, the coefficient of determination indicates "how much of the variation in the dependent variable is affected by the independent variable and how much of the remaining changes in the dependent variable are related to other factors".

The determination coefficient is always between 0 and $100 \%$. $0 \%$ indicates that the model does not describe the response data variability around its mean, and $100 \%$ indicates that the model describes all response data variability around its mean.

Eq. (3) is used to calculate this coefficient:

$$
R^{2}=1-\frac{S S_{r e s}}{S S_{t o t}}
$$

In Eq. (4), $S S_{\text {res }}$ and $S S_{\text {tot }}$ are derived as:

$$
S S_{t o t}=\sum_{i}\left(y_{i}-\bar{y}\right)^{2}
$$

In the equations above, $\bar{y}$ is the mean of the main data which is derived by $\bar{y}=\sum_{i} y_{i}$. The most important innovations of this paper are as follows:

1. A detailed analysis of parameters affecting the consumption and price fluctuations in the Iran market;

2. The proposed system is completely automated and many assumptions, which were empirically calculated and considered by experts, are eliminated. The results obtained in the next season show that compared to human experts who usually make mistakes due to large amounts of data, the proposed 
system has very good performance and works better and more accurately;

3. k-means clustering method is used in the proposed method for calculating the number of similar methods. The proposed method uses a new method based on the Fisher algorithm to automatically calculate the number of clusters of this method;

4. A hybrid network is used to analyze data that is different in nature. In fact, a unique network is trained for near-nature inputs;

5. The initial network weights is a fundamental challenge in learning neural networks. Therefore, the initial evolutionary genetic algorithm is used to improve the initial weights, so as to select the initial weights well. It should be noted that it could be also used for other evolutionary algorithms such as genetics or bee colony algorithms. Each of these methods can have a proper function in the proposed method. This method is used for its simplicity of implementation and its application in many studies in recent years;

6. The lack of precise information is the main challenge in the price forecast. Therefore, the experiments indicated that if there was no information on a region, the proposed method still would have good performance;

7. Compared with other methods introduced in recent years, the proposed method has better performance.

\section{Results}

Table 1 shows the structure of the temperature network for separate seasons. In this table, each year is predicted separately.

It is noteworthy that for values related to the number of neurons, the number of hidden layers and thresholds are derived from the validation set. In this

Table 1. MLP network configuration parameters for input temperature and load consumption.

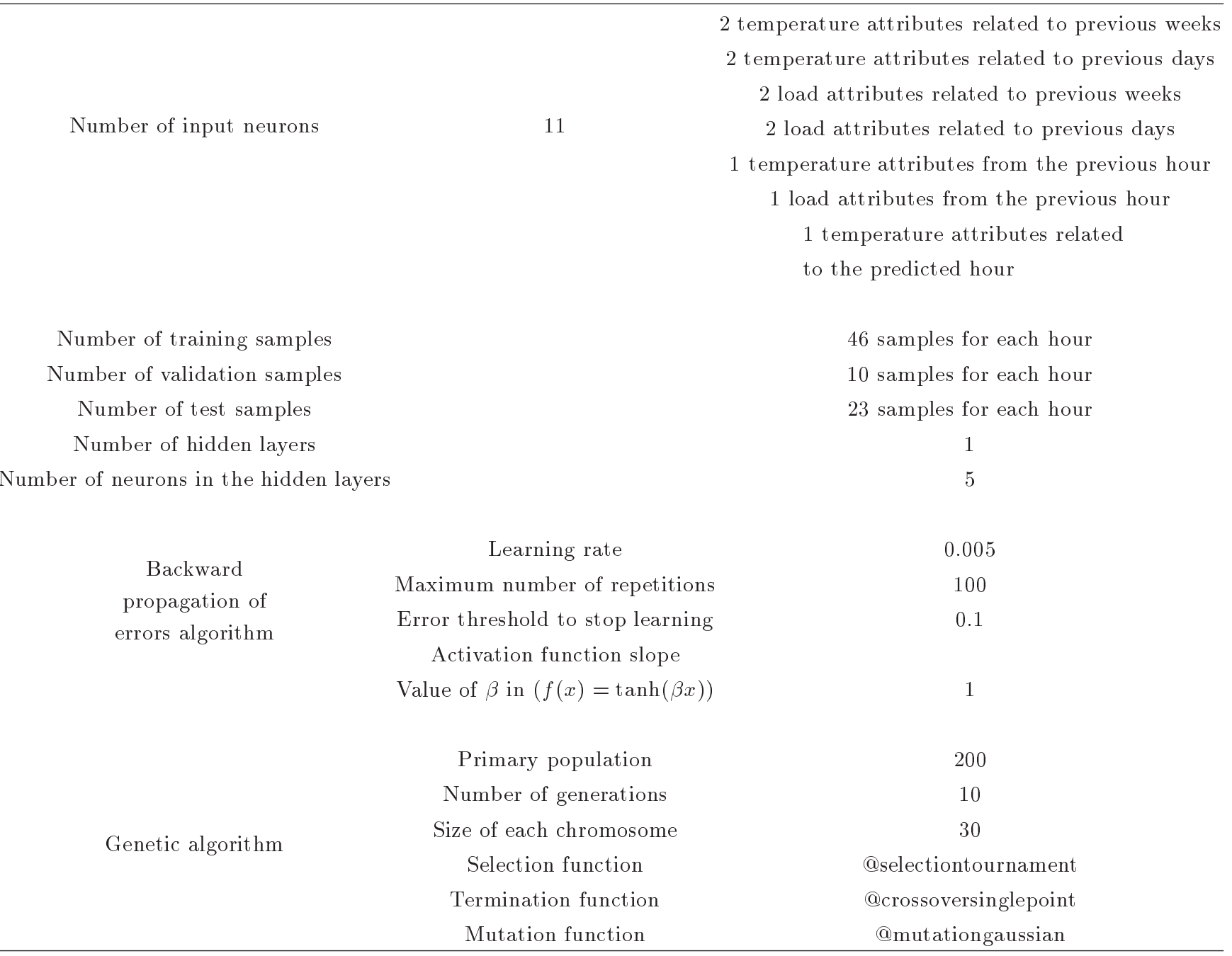


vein, a sample of the results obtained in the summer season is shown in Figure 2 for these parameters. As shown in Figure 2(a), the best number of neurons is 5 . The number of hidden layers according to Figure 2(b) and the threshold according to Figure 2(c) was considered 0.1. All results for subsequent experiments are obtained by validated data.

A structure similar to Table 1 is used to design a network related to price. The number of price attributes in the present structure is one, which is less than the inputs in Table 1, which deals with prediction

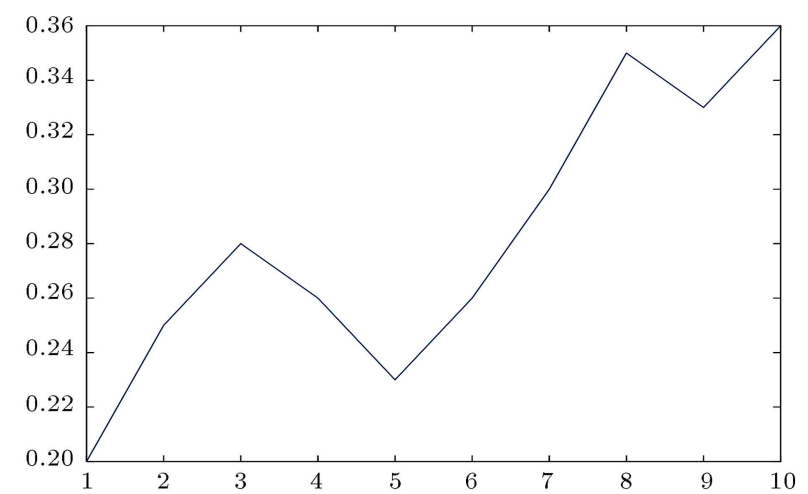

(a)

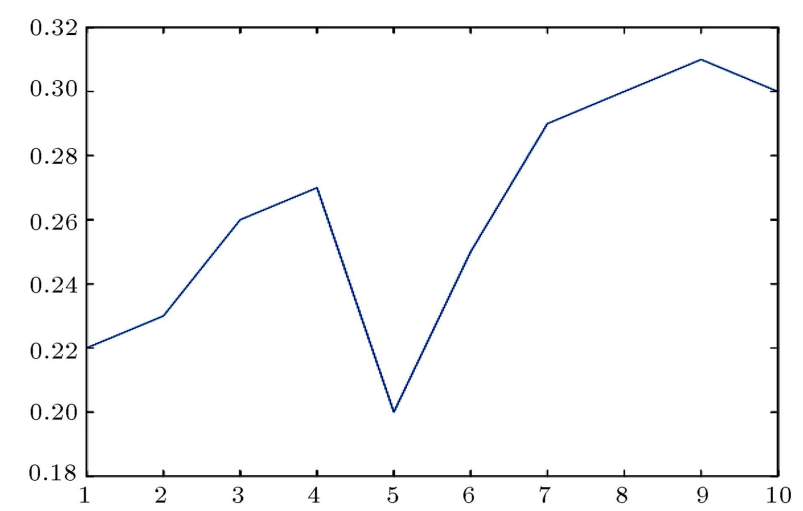

(b)

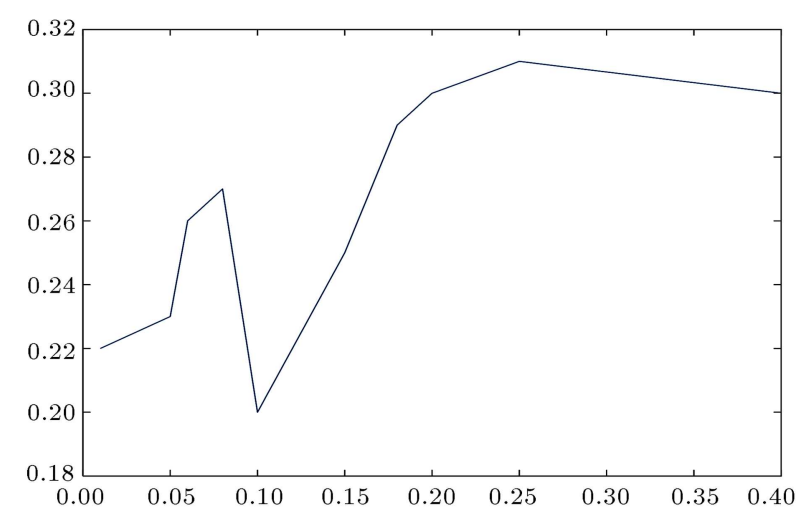

(c)

Figure 2. Estimation of parameters used for the neural network based on the validation data set: (a) Number of neurons, (b) number of hidden layers, and (c) threshold. hour. In this network, for the load consumed in the previous hour (except for $1 \mathrm{am}$ ), the prediction from the previous stage is used. Essentially, the prediction for the current hour is used as the input for the next hour.

The structure of the MLP neural network combining two MLP networks is shown in Table 2.

In this research, in order to analyze each season separately, separate tests are designed for each season. However, it should be noted that the main problem with this type of data is related to summer, so although the proposed method shows appropriate results for all seasons, the focus should be on this point. the main issue with this type of data concerns the summer season, thus it should be focused on even though the proposed method exhibited appropriate results for all seasons. The results of the training and testing of load data for the summer season and autumn season, which represent the other two seasons (due to the similarity of consumption in these seasons), are shown. Also, a comparison between the proposed hybrid method and the MLP method is presented. This chart shows the error rate for the autumn season of the year 2016 in Iran.

\subsection{Summer season}

Based on Figure 3, the slope of Fisher criterion changes in the $k$-means algorithm is not evident from six clusters to seven clusters, thus six clusters are automatically considered for the outputs of this particular hour. In the next step, a neural network for temperature and price are considered for each cluster, which is finally combined by another perceptron network. In the training of these networks, the backward propagation of errors algorithm is used but in the first step, in order to select appropriate initial weights, the genetic algorithm is used instead of random selection. Figure 4 shows the steps involved in implementing the training stage of a network using the genetic algorithm. As previously mentioned, the training number is kept low to increase computing speed. The weights obtained from this method are considered as the initial weights in the network. It should be noted that a genetic

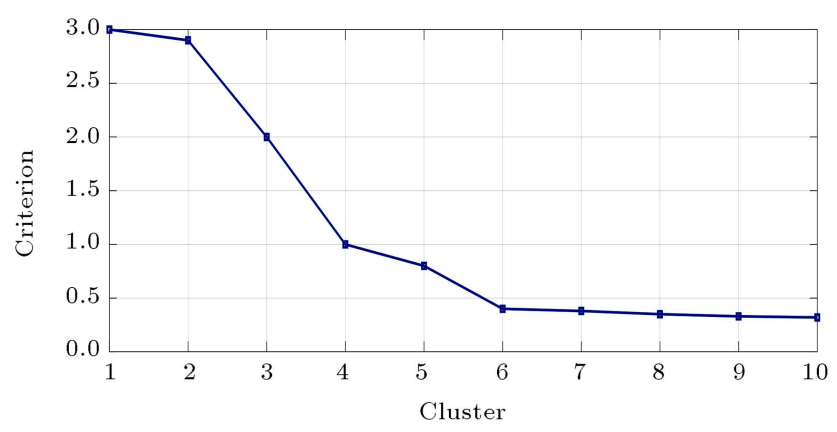

Figure 3. The rate of change in Fisher criterion based on the number of clusters. 
Table 2. Network configuration parameters for multi-layer perceptron network in order to combine networks.

Number of input neurons 2

1 output attribute related to the MLP network with price input 1 output attribute related to the MLP network with temperature input

46 samples for each hour
10 samples for each hour
23 samples for each hour

1

3

Number of neurons in hidden layer

Learning rate

Maximum number of repetitions

Backward propagation of errors algorithm

Error threshold to stop learning

Activation function slope

Value of $\beta$ in $(f(x)=\tanh (\beta x))$

1

Primary population

Number of generations

10

Size of each chromosome

Genetic algorithm

Selection function

Termination function

Mutation function

9

@selectiontournament

@crossoversinglepoint @mutationgaussian

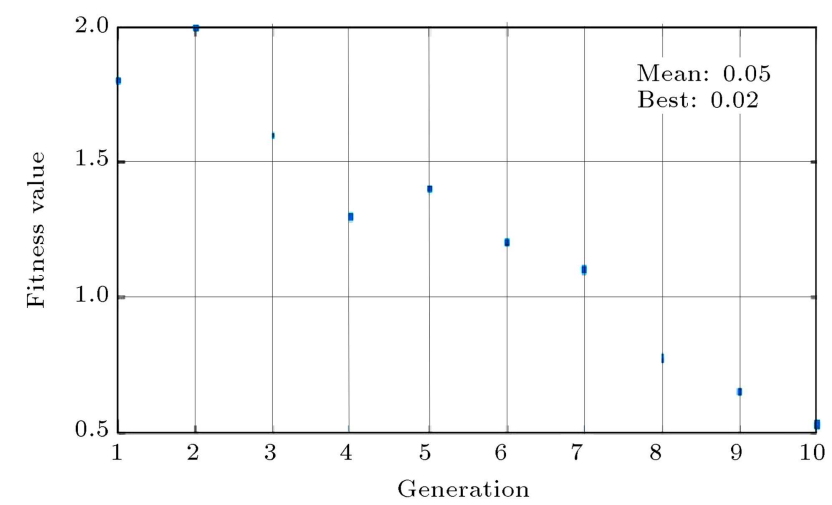

Figure 4. Obtained fitted values using the genetic algorithm in 10 repetitions.

algorithm should be implemented for each MLP neural network. Lastly, the network is trained and tested according to the previous steps. This chart shows the error rate for summer days of the year 2016 in Iran.

The results for the training data for the summer season are shown in Figure 5. Figure 5(a) presents the results of the network training using MLP and Figure 5(b) presents the results of combining the MLP and SOM network. As depicted in this figure, the proposed method exhibited superior performance compared to typical methods in the training stage.
The results for the experiment series for the summer season are shown in Figure 6. Figure 6(a) shows the results of using MLP and Figure 6(b) shows the result of combining the MLP and SOM network, which is similar to the training phase in the proposed method, showing excellent performance in the experimental phase.

\section{Conclusion}

In this paper, a dynamic method was proposed to predict electricity prices. In the proposed method, artificial intelligence-based algorithms were used. Data variances and analysis were the main focus to achieve more accurate predictions. As mentioned earlier, there are many factors that affect price prediction, but it is a difficult task to obtain all the necessary information in the current state of the world. Therefore, it was aimed to utilize a system that is compatible with existing data. A hybrid approach based on neural networks was used in the proposed method. Since similar data from different days at different hours may produce identical results, similar data from the training and experiment stages are used as input. Thus, the unsupervised SOM neural network was used to cluster samples.

It should be noted that upon analysis, extracted 


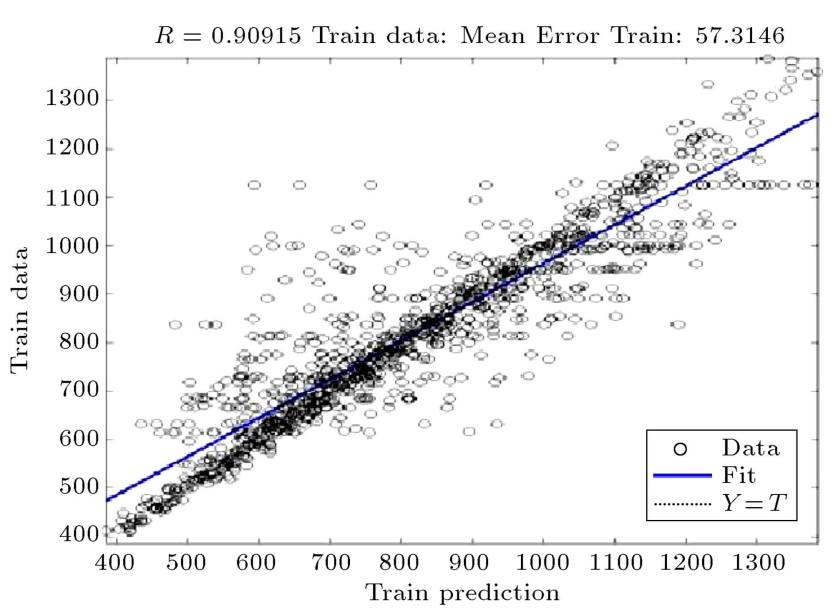

(a)

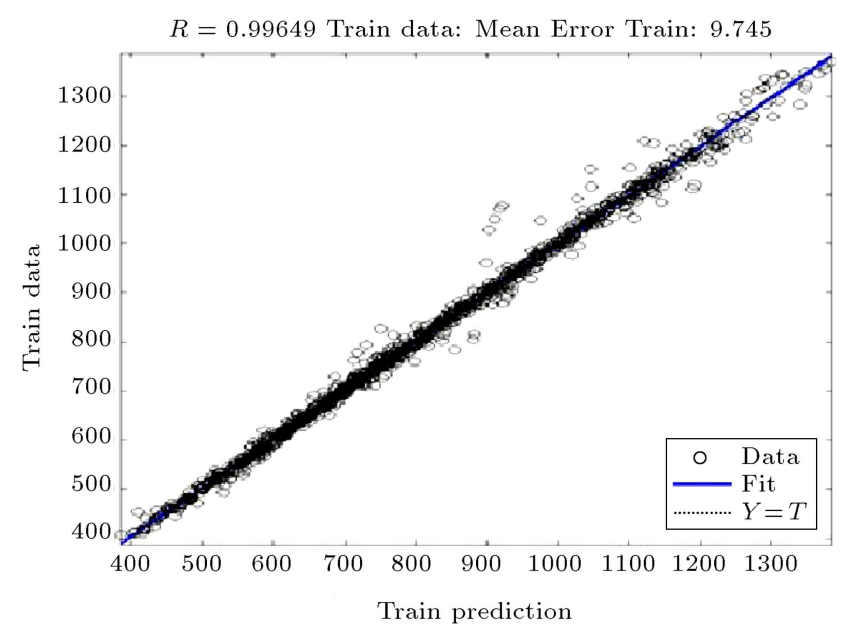

(b)

Figure 5. Results of the training stage for the summer season: (a) MLP network and (b) MLP and SOM hybrid network.

data of different seasons exhibited different performances. In the same vein, analyzes related to different seasons were conducted separately. For the next stage, similar data were used based on the day, prior to the analysis of the neural network input data. According to this analysis, the data related to the price and the data related to the consumed load and temperature were of a different nature.

This research uses two multilayer perceptron neural networks to make predictions. Essentially, in the proposed method, a network is used for training data related to temperature, and another network is used for training data related to price. According to the obtained results, data analysis using different networks is influential on the accuracy of the proposed method. In the training phase, the appropriate selection of initial weights is vital in training neural networks. Thus, for improved training of the proposed network, an evolutionary search method is used to derive initial weights. Finally, the proposed networks were combined

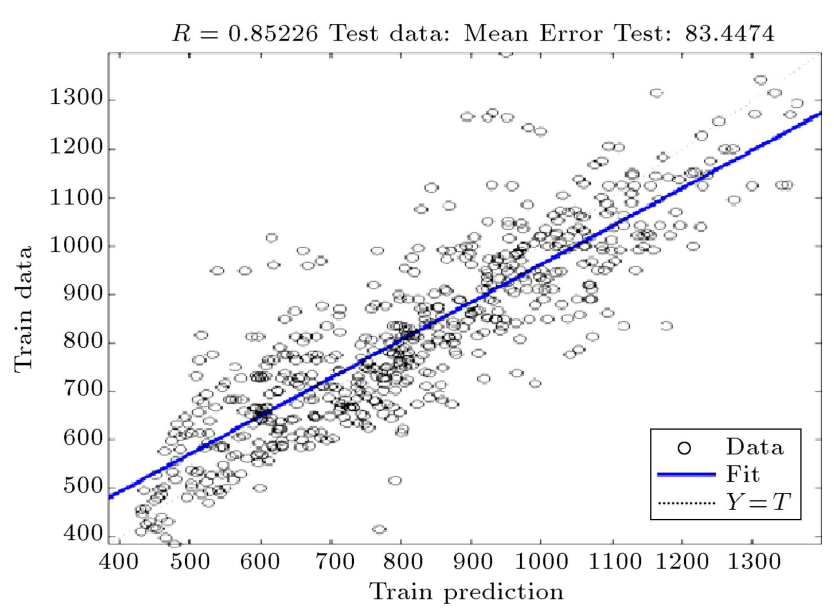

(a)

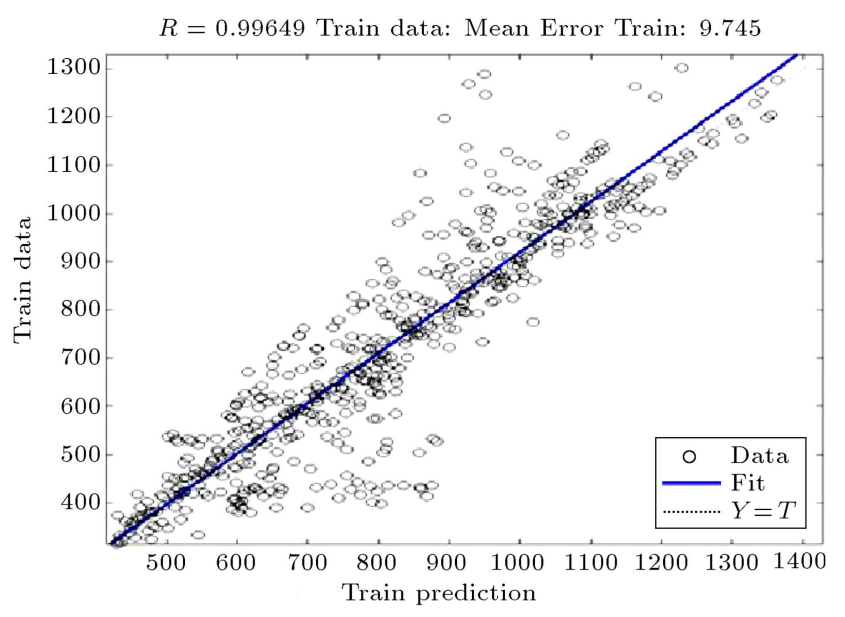

(b)

Figure 6. Results of the experiment stage for the summer season: (a) MLP network and (b) MLP and SOM hybrid network.

with another MLP neural network and the results indicated the improved performance of the proposed method compared to other methods.

Some of the most significant innovations presented in this study are listed below:

1. An accurate analysis of the parameters affecting consumption and price fluctuations in the Iranian market is presented. In Iran, Mazandaran province was selected due to its atmospheric volatility, and according to experts, the province is one of the most challenging regions in the country;

2. The proposed method is completely automated and many of the empirically derived preconditions set by experts have been eliminated. The obtained results indicate that the proposed method exhibits favorable performance and is more accurate than the results obtained by a human expert who makes several mistakes due to the high volume of data;

3. In the proposed method, the k-means clustering 
method was used to calculate the number of similar methods. In this method, a novel method based on Fisher algorithm is used to automatically calculate the number of utilized clusters;

4. A hybrid network is used to analyze data that are of different natures. A separate network is trained for each input of data that is essentially close to the network. Finally, the outputs of the networks are combined;

5. One of the fundamental challenges of training neural networks is the allocation of initial weights. In this regard, an evolutionary genetic algorithm is used to improve the initial weights so that the initial weights are appropriately selected;

6. One of the main challenges of the prediction of prices is the lack of accurate information. In this regard, the conducted experiments showed that if there is no information for a particular region, the proposed method still exhibits favorable performance;

7. Compared with other methods proposed in recent years, the proposed method exhibits superior performance.

\section{References}

1. Lin, L., Zhou, C., Saritporn, V., Shen, X., and Dong, M. "Opportunities and challenges for biodiesel fuel", Appl Energy, 88(4), pp. 1020-31 (2011).

2. Dhinesh, B., Raj, Y., Ambrose, M., Kalaiselvan, C., and KrishnaMoorthy, R. "A numerical and experimental assessment of a coated diesel engine powered by high-performance nano biofuel", Energy Convers Manage, 171, pp. 815-24 (2018).

3. Vigneswaran, R., Annamalai, K., Dhinesh, B., and Krishnamoorthy, R. "Experimental investigation of unmodified diesel engine performance, combustion and emission with multipurpose additive along with waterin diesel emulsion fuel", Energy Convers Manage X, 172, pp. 370-380 (2018).

4. Dhinesh, B. and Annamalai, M. "A study on performance, combustion and emission behaviour of diesel engine powered by novel nano nerium oleander biofuel", Journal of Cleaner Production, 196, pp. 74-83 2018.

5. Noboru, N., Atsushi, I., Yutaka, T., Makoto, A. "Lifecycle emission of oxidic gases from power-generation systems", Appl Energy, 68(2), pp. 215-227 (2001).

6. Sánchez de la Nieta, A.A., González, V., and Contreras, J. "Portfolio decision of short-term electricity forecasted prices through stochastic programming", Energies, 9(12), p. 1069 (2016).

7. Najafi, A., Falaghi, H., Contreras, J., and Ramezani, M. "Medium-term energy hub management subject to electricity price and wind uncertainty", Appl Energy, 168, pp. 418-33 (2016).
8. Yang, P., Tang, G., and Nehorai, A. "A game-theoretic approach for optimal time-of-use electricity pricing", IEEE Trans Power Syst, 28(2), pp. 884-92 (2013).

9. Wang, D., Luo, H., Grunder, O., Lin, Y., and Guo, H. "Multi-step ahead electricity price forecasting using a hybrid model based on two-layer decomposition technique and bp neural network optimized by firefly algorithm", Appl Energy, 190, pp. 390-407 (2017).

10. Yang, Z., Ce, L., and Lian, L. "Electricity price forecasting by a hybrid model, combining wavelet transform, arma and kernel-based extreme learning machine methods", Appl Energy, 190, pp. 291-305 (2017).

11. Abedinia, O., Amjady, N., Shafie-khah, M., and Catalão, J.P.S. "Electricity price forecast using combinatorial neural network trained by a new stochastic search method", Energy Convers Manage, 105, pp. 642-654 (2015).

12. Lago, J., Ridder, F.D., Vrancx, P., and Schutter, B.D. "Forecasting day-ahead electricity prices in Europe: the importance of considering market integration", Appl Energy, 211, pp. 890-903 (2018).

13. Weron, R. "Electricity price forecasting: a review of the state-of-the-art with a look into the future", Int $J$ Forecast, 30(4), pp. 1030-81 (2014).

14. Lago, J., Ridder, F.D., and Schutter, B.D. "Forecasting spot electricity prices: deep learning approaches and empirical comparison of traditional algorithms", Appl Energy, 221, pp. 386-405 (2018).

15. Wang, J.Z., Liu, F., Song, Y.L., and Zhao, J. "A novel model: dynamic choice artificial neural network (DCANN) for an electricity price forecasting system", Appl Soft Comput, 48, pp. 281-97 (2016).

16. Bento, P.M.R., Pombo, J.A.N., Calado, M.R.A., and Mariano, S.J.P.S. "A bat optimized neural network and wavelet transform approach for short-term price forecasting", Appl Energy, 210, pp. 88-97 (2018).

17. Ghasemi, A., Shayeghi, H., Moradzadeh, M., and Nooshyar, M. "A novel hybrid algorithm for electricity price and load forecasting in smart grids with demandside management", Appl Energy, 177, pp. 40-59 (2016).

18. Mirakyan, A., Meyer-Renschhausen, M., and Koch, A. "Composite forecasting approach, application for nextday electricity price forecasting", Energy Econ, 66, pp. 228-237 (2017).

19. Sanjeev Kumar Aggarwal, Lalit Mohan Saini, Ashwani Kumar, "Electricity price forecasting in deregulated markets: A review and evaluation", International Journal of Electrical Power $\& 5$ Energy Systems, 31, pp. 13-22 (2009).

20. Panapakidis, I.P. and Dagoumas, A.S. "Day-ahead electricity price forecasting via the application of artificial neural network based models", Appl Energy, 172, pp. 132-51 (2016). 
21. Sandhu, H.S., Fang, L.P., and Guan, L. "Forecasting day-ahead price spikes for the Ontario electricity market", Electr Pow Syst Res, 141, pp. 450-459 (2016).

22. Ortiz, M., Ukar, O., Azevedo, F., and Múgica, A. "Price forecasting and validation in the Spanish electricity market using forecasts as input data", Electr Pow Energy Syst, 77, pp. 123-127 (2016).

23. Keles, D., Scelle, J., Paraschiv, F., and Fichtner, W. "Extended forecast methods for day-ahead electricity spot prices applying artificial neural networks", Appl Energy, 162, pp. 218-230 (2016).

24. Singh, N., Mohanty, S.R., and Shukla, R.D. "Short term electricity price forecast based on environmentally adapted generalized neuron", Energy, 125, pp. 127-139 (2017).

25. Itaba, S. and Mori, H. "A fuzzy-preconditioned GRBFN model for electricity price forecasting", Proc Comput Sci, 114, pp. 441-448 (2017).

26. Wang, D.Y., Luo, H.Y., Grunder, O., et al. "Multi-step ahead electricity price forecasting using a hybrid model based on two-layer decomposition technique and BP neural network optimized by firefly algorithm", Appl Energy, 190, pp. 390-407 (2017).

27. Tang, L., Yu, L., and He, K. "A novel datacharacteristic-driven modeling methodology for nuclear energy consumption forecasting", Appl Energy, 128, pp. 1-14 (2014).

28. Chai, J., Zhang, Z.Y., Wang, S.Y., Lai, K.K., and Liu, J. "Aviation fuel demand develop in China", Energy Econ, 46, pp. 224-35 (2014).

29. Diongue, A.K., Guegan, D., and Vignal, B. "Forecasting electricity spot market prices with a k-factor GIGARCH process", Appl Energy, 86, pp. 505-510 (2009).

30. Girish, G.P. "Spot electricity price forecasting in Indian electricity market using autoregressive-GARCH models", Energy Strateg Rev, 11, pp. 52-57 (2016).

31. Zhang, J.L. and Tan, Z.F. "Day-ahead electricity price forecasting using WT, CLSSVM and EGARCH model", Electr Pow Energy Syst, 45, pp. 362-368 (2013).

32. Zhang, Y., Li, C., and Li, L. "Electricity price forecasting by a hybrid model, combining wavelet transform, ARMA and kernel-based extreme learning machine methods", Appl Energy, 190, pp. 291-305 (2017).

33. Yan, X. and Chowdhury, N.A. "Mid-term electricity market clearing price forecasting: a hybrid LSSVM and ARMAX approach", Electr Pow Energy Syst, 53, pp. 20-6 (2013).

34. Zhu, B.Z. and Wei, Y.M. "Carbon price prediction with a hybrid ARIMA and least squares support vector machines methodology", Omega, 41, pp. 517-24 (2013).
35. He, K.J., Yu, L., and Tang, L. "Electricity price forecasting with a BED (Bivariate EMD Denoising) methodology", Energy, 91, pp. 601-609 (2015).

36. Qiu, X.H., Suganthan, P.N., and Amaratunga, G.A.J. "Short-term electricity price forecasting with empirical mode decomposition based ensemble kernel machines", Proc Comput Sci, 108, pp. 1308-1317 (2018).

\section{Biographies}

Seyed Mojtaba Kavoosi Davoodi is a $\mathrm{PhD}$ in the Industrial Engineering Department at the Islamic Azad University, Science and Research Branch in Tehran, Iran. He received the BA degree from the Mazandaran University of Science and Technology (MUST), the MS degree from the Industrial Engineering Department, Islamic Azad University, Science and Research Branch, Tehran, Iran. His research interests include engineering management, and strategic management, decision making, Fuzzy systems, computational modeling, data envelopment analysis, neural network, and optimization techniques.

Seyed Esmaeil Najafi is an Assistant Professor of the Industrial Engineering Department at the Islamic Azad University, Science and Research Branch in Tehran, Iran. He received the BA degree from the Power and Water University of Technology (PWUT), the MS degree from the Islamic Azad University, and the PhD degree in Industrial Engineering from the Science and Research Branch, Tehran, Iran. He is currently an Assistant Professor with the Industrial Engineering Department, Islamic Azad University, Science and Research Branch, Tehran, Iran. His research interests include computational modeling, decision making, data envelopment analysis, engineering management, and strategic management. His published research articles appear in the Journal of Intelligent and Fuzzy Systems, the Mathematical Problems in Engineering, and the International Journal of Data Envelopment Analysis.

Farhad Hosseinzadeh Lotfi is currently a Full Professor in Mathematics at the Science and Research Branch, Islamic Azad University (IAU), Tehran, Iran. In 1991, he received his undergraduate degree in Mathematics at Yazd University, Yazd, Iran. He received his MSc in Operation Research at IAU, Lahijan, Iran in 1995 and PhD in Applied Mathematics (O.R.) at IAU, Science and Research Branch, Tehran, Iran in 1999. His major research interests are operation research and data envelopment analysis. He has been Advisor and Co-advisor of 46 and $31 \mathrm{PhD}$ Dissertations, respectively. He has published more than 300 scientific and technical papers in leading scientific journals, including the European Journal of Operational Research, 
Computers and Industrial Engineering, Journal of the Operational Research Society, Applied Mathematics and Computation, Applied Mathematical Modelling, Mathematical and Computer modelling, and Journal of the Operational Research Society of Japan, among others. He is the Editor-in-Chief and member of the editorial board of the Journal of Data Envelopment Analysis and Decision Science. He is also manager and member of the Editorial Board of the International Journal of Industrial Mathematics.
Hossein Mohammadian Bisheh received the BSc degree in Electrical Engineering from the Sharif University of Technology, Tehran, Iran in 1971, and the MSc and $\mathrm{PhD}$ degrees both in Industrial Engineering from Mazandaran University of Science and Technology, Babol, Iran in 1996 and 2009. He is an Assistant Professor at Mazandaran University of Science and Technology, Babol, Iran. His research interests are power system planning and control, energy management systems, and optimization techniques. 